\title{
Adverse Events During Neoadjuvant Chemotherapy for Muscle Invasive Bladder Cancer
}

\author{
Antti P. Salminen ${ }^{\mathrm{a}, *}$, Ileana Montoya Perez ${ }^{\mathrm{b}}$, Riku Klén $^{\mathrm{c}, \mathrm{d}}$, Otto O. Ettala ${ }^{\mathrm{a}}$, Kari T. Syvänen ${ }^{\mathrm{a}}$, \\ Laura L. Elo ${ }^{\mathrm{c}}$ and Peter J. Boström ${ }^{\mathrm{a}}$ \\ ${ }^{a}$ Department of Urology, University of Turku and Turku University Hospital, Turku, Finland \\ ${ }^{\mathrm{b}}$ Department of Information Technology, University of Turku, Turku, Finland \\ ${ }^{\mathrm{c}}$ Turku Bioscience Centre, University of Turku and Åbo Akademi University, Turku, Finland \\ ${ }^{\mathrm{d}}$ Turku PET Centre, Turku, Finland
}

\begin{abstract}
.
PURPOSE: Neoadjuvant chemotherapy (NAC) improves survival in muscle invasive bladder cancer (MIBC). Rate of adverse events (AE) have been reported only in randomized clinical trials (RCT). Purpose was to evaluate incidence, type, and risk factors of AEs during NAC treatment in a population-based setting.

MATERIAL AND METHODS: The Finnish national cystectomy database covering years 2008-2014 was utilized. NAC associated AEs were reported by Common Terminology Criteria for Adverse Events (CTCAE) v.5.0. AEs during NAC in five-tier severity scale was the outcome measure. Spearman correlation between AEs and 22 clinical variables were calculated. $P$-values were corrected for multiple testing by controlling false discovery rate (FDR) with Benjamini-Hochberg method.

RESULTS: Thirty-one percent of MIBC patients were assigned to NAC. Final analysis included 229 NAC patients representing 30\% of radical cystectomy (RC) population. Majority (88\%) received cisplatin-gemcitabine. 105 patients (46\%) had no AEs. 124 patients (54\%) had 168 AEs in total. Severe events (CTCAE grade 3-5) were documented in 31\% of patients and one $(0.4 \%)$ died. In five patients $(2.1 \%) \mathrm{RC}$ was not performed due to the AE. Of the severe AEs, hematological were most common, followed with cardiac, vascular and urinary tract as most commonly affected organ systems. The number of chemotherapy cycles was the only variable significantly associated with AEs. Severe AEs occurred already during or after the first cycle of NAC leading to early termination.

CONCLUSION: NAC is generally well tolerated, but poses a considerable risk for adverse events. This is the first study to evaluate AEs caused by NAC in real life scenario on population level.
\end{abstract}

Keywords: Neoadjuvant chemotherapy, Bladder cancer, adverse events

\section{INTRODUCTION}

Cisplatin-based neoadjuvant chemotherapy (NAC) has an integral role in treatment of muscle invasive bladder cancer (MIBC) with 5\% absolute improvement in cancer specific survival [1-3]. If NAC is

\footnotetext{
*Correspondence to: Antti Salminen, MD, Department of Urology, University of Turku and Turku University hospital, Kiinamyllynkatu 4-8, 20520 Turku, Finland. Tel.: +358 23130000; Fax: +358 2 3132284; E-mail: antti.salminen@tyks.fi.
}

tolerated, it does not increase post-operative complications [4-6]. Platinum compounds (cisplatin, carboplatin) have many potential side-effects [7]. Cardiac, vascular, pulmonary and urinary systems may be damaged and septic complications due to neutropenia are a major concern. Platinum is ototoxic and peripheral neuropathy has been identified as a potential side effect [7]. Thromboembolic events are common, but seldom eliminate the execution of radical cystectomy $(\mathrm{RC})$ [8]. 
The rationale in multimodality treatment of MIBC is to optimize cancer control without overtreatment and with minimal risk of NAC associated adverse events (AEs). Tolerability and toxicity are best reported in randomized clinical trials (RCT) [2, 9-11]. In RCTs, the reported mortality rates during NAC differ from 0 to $1 \%$ [10]. However, it should be noted that in RCTs eligible patients are highly selected, and this does not necessarily correlate to "real-life scenario". There are no population based studies reporting NAC related AEs in MIBC.

In Finland NAC was introduced first in 2008 and more widespread usage was initiated in 2011. The national policy favors cisplatin-gemcitabine (CG) with differences concerning number of cycles or interval between cycles among hospitals treating MIBC. Nevertheless, the usage of NAC has steadily gained popularity but critical evaluation of NAC associated AEs has not been performed. Outside RCTs this subject has not been thoroughly evaluated before. Therefore, to gain knowledge in real life setting, we conducted a retrospective national study to evaluate AEs caused by NAC.

\section{MATERIAL AND METHODS}

The study was approved by the ethical committee of the hospital district of southwestern Finland (ETMK: 4/1802/2015). Due to the retrospective nature of data collection, a signed informed consent were not obtained. The study population was derived from the Finnish national cystectomy database which is described in detail previously [6]. In short, the database is an internet-based data collection platform, where essential clinic-pathological data of all RC patients operated between 2005-2015 was collected retrospectively from all 16 different Finnish hospitals performing these operations during the observation period. The patients in the cystectomy database were identified using International Statistical Classification of Diseases and Related Health Problems (ICD-10) codes (C67.*) and surgical procedure coding (Nordic Classification of Surgical Procedures). Details of NAC included regimen, number of cycles, AEs and clinical response. To ensure that all patients who entered NAC treatment during the observation period were included, an additional query was sent to all participating centers to recognize NAC patients, to whom RC was not performed for any reason. By comparing oncological and urological MIBC patient charts we were able to identify seven patients that never had RC after initiation of NAC.
NAC associated AEs were reported by Common Terminology Criteria for Adverse Events (CTCAE) v. 5.02018 [12]. The CTCAE is a five-tiered scale, where 0 means no adverse events, 1-2 minor events, 3 severe events, 4 permanent damage and 5 means death. This grading system divides events to 26 different classes, which are further divided to sub-groups so that events can be classified in a very detailed manner. Only events during neoadjuvant chemotherapy prior to cystectomy were documented, since later complications are contaminated with RC involvement and the role of NAC in postoperative complications has already been analyzed in the same population [6] Since pain may be associated with cancer itself and it was not systematically documented, we chose not to consider pain as an AE caused by NAC.

In Finland multidisciplinary approach of MIBC treatment consist of urologist, medical oncologist, pathologist and radiologist and chemotherapy is given under supervision of medical oncologist. Data was collected by urologists. To further substantiate grade and quantity of these events, all patients and events were individually reviewed by responsible author for a secondary verification.

Spearman correlation between AEs and 22 clinical variables were calculated. $P$-values were corrected for multiple testing by controlling false discovery rate (FDR) with Benjamini-Hochberg method [13]. Statistical analysis was done using R [14].

\section{RESULTS}

We were able to identify 239 patients who were assigned to NAC treatment during the observation period. This represents $31 \%$ of the MIBC patients scheduled to RC. Two patients refusing RC and receiving radiotherapy as well as eight patients with prematurely terminated NAC due to disease progression were excluded. After exclusion, total number of patients in final analysis was 229. Basic characteristics of the study population are presented in Table 1 . Study population represents typical Caucasian MIBC population: $80 \%$ males, mean age of 65 years, slightly obese with BMI of $26 \mathrm{~kg} / \mathrm{m}^{2}$. Mean number of chemotherapy cycles was three, and cisplatingemcitabine (CG) was the preferred regimen (90\%). Seven percent had carboplatin-gemcitabine and 3\% other regimens due to small cell or squamous cell bladder carcinomas. Sixty five percent of patients were considered either having clinical response or stable disease for NAC. Thirty seven percent were 
Table 1

Basic characteristics of the 229 patients in the study population

\begin{tabular}{llll}
\hline Variable & Description & & \\
\hline Gender & Male & $\mathrm{N}(\%)$ & $189(83)$ \\
& Female & & $40(17)$ \\
Age & Years & Mean (range) & $65(44-81)$ \\
$\mathrm{BMI}^{1}$ & $\mathrm{Kg} / \mathrm{m}^{2}$ & Mean (range) & $26(16-39)$ \\
$\mathrm{ASA}^{2}$ & $1-2$ & $\mathrm{~N}(\%)$ & $96(42)$ \\
& $3-4$ & & $105(46)$ \\
& Unknown & & $28(12)$ \\
$\mathrm{ACCI}^{3}$ & & Mean (range) & $4(1-10)$ \\
$\mathrm{NAC}^{4}$ modality & Cis - gem & $\mathrm{N}(\%)$ & $205(90)$ \\
& Car -gem & & $16(7)$ \\
& Other & & $8(3)$ \\
$\mathrm{Cycles}^{2}$ & number & $\mathrm{N}(\%)$ & $111(48)$ \\
$\mathrm{Clinical}^{2}$ response to NAC & Yes & & $42(18)$ \\
& No (stable disease) & & $9(4)$ \\
& Progression & & $31(13)$ \\
& Lack of tolerance & & $36(16)$ \\
\hline
\end{tabular}

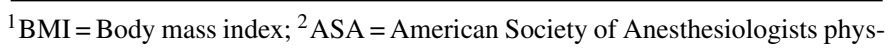
ical status classification ${ }^{3} \mathrm{ACCI}=$ Age adjusted Charlson comorbidity index; ${ }^{4} \mathrm{NAC}=$ Neoadjuvant chemotherapy; ${ }^{5}$ Clinical response was evaluated with Computed tomography after 2 cycles of chemotherapy.

Table 2

Distribution of adverse events during neoadjuvant chemotherapy based on Common Terminology Criteria of Adverse Events (CTCAE-classification)

\begin{tabular}{lcc}
\hline $\begin{array}{l}\text { Complication } \\
\text { severity grade }\end{array}$ & $\begin{array}{c}\text { No of patients } \\
\text { (Ntot) }=229\end{array}$ & $\%$ \\
\hline 0 & 105 & 46 \\
1 & 17 & 7 \\
2 & 31 & 13 \\
3 & 49 & 21 \\
4 & 26 & 11 \\
5 & 1 & 0.5 \\
\hline
\end{tabular}

Only the most severe adverse event of each patient has been selected despite one patient may have multiple events.

never smokers, and $63 \%$ of patients were either former $(33 \%)$ or current $(30 \%)$ smokers. Smoking history was not documented in $19 \%$ of patients. For those who were either former or current smokers, mean quantity of smoking was 36 pack years, range (4-100).

Table 2 presents overall distribution of AE severity by CTCAE grade on patient level. Total number of all AEs was 168 in 124 (54\%) patients. Death occurred in one patient $(0.4 \%)$, severe (grade 3-4) events were seen in $32 \%$ of patients and in $20 \%$ of patients AEs were considered as minor (grade 1-2). One hundred and five patients (46\%) had no NAC associated AEs.

A more detailed distribution of the most severe AEs by organ system is presented in Table 3. Among 27 patients with severe AEs, hematologic AEs were the most common (33\%). Cardiovascular events were seen on $30 \%$ of patients, $15 \%$ had permanent damage to urinary system, and one patient (3\%) had liver failure. In 5 patients (19\%) RC could not be executed after NAC.

Correlations between the investigated variables and AEs are presented in Table 4. After FDR correction, the number of NAC cycles was negatively correlated with the rate of AEs. Severe AEs occurred already during or after the first cycle of NAC leading to early termination, whereas, in patients tolerating several cycles, AEs were seldom seen. No statistically significant correlation was observed between other variables and the AEs.

\section{DISCUSSION}

Our population-based study demonstrates that $46 \%$ of patients tolerated NAC well without AEs. Severe AEs were documented in $30 \%$ of patients and minor events were seen on $20 \%$ of patients. Finally, $2.5 \%$ of patients either died $(0.4 \%)$ or were unfit (2.1\%) for RC after NAC. To our best knowledge, this is the first study reporting AEs during NAC on population based setting.

In RCTs the reported mortality during NAC is $0-1 \%$. [2, 9-11, 15]. Our mortality rate of $0.4 \%$ is therefore comparable to the reported rates. Although death is rare, NAC poses patients to other clinically significant morbidities, especially to hematological 
Table 3

Distribution of most severe adverse events (CTCAE Grade 4-5) on patient level by organ system

\begin{tabular}{lccl}
\hline Organ system & $\begin{array}{c}\text { Quantity } \\
\text { Ntot }=27\end{array}$ & $\%$ & Description \\
\hline Hematologic & 9 & 33 & Cytopenia, septic infection \\
Cardiac & 4 & 15 & Infarct, failure, myopericarditis, death \\
Vascular & 4 & 15 & Pulmonary embolism (2), critical ischemia (2) \\
Urinary & 4 & 15 & Kidney failure (3), obstruction and septic infection (1) \\
Hepatobiliary & 1 & 3 & Liver failure \\
Non-specific & 5 & 19 & Patient unfit for surgery after NAC \\
\hline
\end{tabular}

Table 4

Correlation between adverse event occurring during neoadjuvant chemotherapy and 22 clinical variables

\begin{tabular}{lcc}
\hline Variable & $\begin{array}{c}\text { Correlation } \\
\text { coefficient }^{1}\end{array}$ & $P$-value \\
\hline No of Cycles & -0.37 & $<0.001$ \\
Renal disease & 0.17 & 0.052 \\
Cerebrovascular disease & -0.13 & 0.205 \\
Rheumatoid disease & -0.13 & 0.229 \\
PAD $^{2}$ & 0.12 & 0.239 \\
Myocardial infarction & -0.11 & 0.257 \\
Congestive heart failure & 0.10 & 0.359 \\
Body mass index & -0.10 & 0.371 \\
Age & 0.06 & 0.442 \\
Height & 0.08 & 0.442 \\
ASA class & 0.07 & 0.442 \\
Smoking pack years & 0.08 & 0.442 \\
Dementia & 0.06 & 0.442 \\
Chronic pulmonary & -0.08 & 0.442 \\
Peptic ulcer & 0.06 & 0.442 \\
Liver disease & -0.07 & 0.442 \\
Leukemia & -0.07 & 0.442 \\
CCI score & -0.06 & 0.470 \\
Solid tumor & -0.03 & 0.747 \\
ACCI & 0.03 & 0.747 \\
Diabetes & -0.01 & 0.860 \\
Weight & 0.00 & 0.960 \\
\hline I & &
\end{tabular}

${ }^{1}$ Correlation coefficient describes the strength of correlation with the adverse event ranging between -1 and 1 . The greater the difference from 0 , the stronger the correlation is. Positive correlation as in Renal disease indicates that it is correlated with high CTCAE. Negative correlation as in No of Cycles suggests that adverse events tend to occur already during first cycles of chemotherapy; adding chemotherapy cycles does not increase risk for AEs. ${ }^{2} \mathrm{PAD}=$ Peripheral arterial disease $;{ }^{3} \mathrm{ASA}=$ American association of anesthesiologists to evaluate fitness for surgical operations; ${ }^{4} \mathrm{CCI}=$ Charlson comorbidity index; ${ }^{5} \mathrm{ACCI}=$ Age adjusted Charlson comorbidity index.

AEs. In a large RCT, hematologic World Health Organization (WHO) grade 3 or 4 AEs were reported on $32.5 \%$ of patients receiving cisplatin, methotrexate and vinblastine (MVC) [10]. In addition, Grossman et al. reported grade 4 hematologic toxicities on $33 \%$ of patients using cisplatin, methotrexate, doxorubicin and vinblastine (MVAC) [2]. In our study grade 4 hematological toxicities were reported only on nine patients $(4 \%)$ of patients. Both of these prospective studies utilized different chemotherapy regimen, either CMV [10] or MVAC [2]. Although CG seems to have better tolerance profile concerning hematologic AEs, the NAC regimen unlikely explains completely this difference. Many RCTs recruited patients over two decades ago and there may be better prophylactic methods to prevent AEs. Specifically, the lower incidence may be due to current standard use of filgrastim during NAC treatment in Finland. Other explanation may be differences in the investigational approach - prospective and retrospective studies cannot be compared directly.

Severe, grade 4 or 5 AEs, are the most concerning. In our study, the radical cystectomy operation was cancelled due to AEs in $2.1 \%$ of patients. In a prospective study by Grossmann et al. the scheduled cystectomy was performed only on $82 \%$ of patients. Reasons for not performing surgery were either medical or other [2]. Possible explanations are patient preference or disease progression as in daily clinics, but details are not described more thoroughly in the publication and this remains speculative. Despite 11 $\%$ of grade 4 toxicities in our study, radical cystectomy could be performed to $97.5 \%$ of patients.

Although chemotherapy is based on platinum, different regimen options are available. A direct comparison between the efficacy of CG or MVAC in NAC setting has been retrospectively evaluated in a study by Fairey et al. [16]. Although the results seem to favor MVAC instead of CG, in a propensity matched approach by Galsky et al., no difference between these two modalities concerning efficacy could be detected [17]. However, neither of the two studies investigated NAC associated AEs. The comparison of toxicity has only been evaluated in metastatic setting. In phase III RCT, von der Maase et al. [18] reported mortality rates of $1 \%$ and $3 \%$ favoring CG over MVAC. In addition, risk-benefit ratio was superior in GC arm compared with MVAC arm. It is however noteworthy to remember that in metastatic $\mathrm{BC}$, only $60 \%$ of patients may be platinum eligible and the results cannot be generalized to NAC patients [19]. 
Correlation between NAC related AEs and dosing are conflicting $[11,17]$. While Iyer et al. found 6 cycles of CG to be safe, Anari et al. had to close their study early due to higher than suspected rate of serious vascular events $[11,20]$. These were both relatively small phase II studies evaluating efficacy and safety. In the study by Iyer et al. dose adjustment were necessary for $39 \%$ of patients and $67 \%$ of patients received the chemotherapy as planned [11]. In the study by Anari et al. 23\% of patients suffered from serious vascular event during NAC, but $94 \%$ of the patients were fit for RC showing that even after grade IV complication surgery can be performed in most cases [20]. In Finland, either three or four cycles of CG within three or four week interval is normally given. Between these two protocols no difference between the rate of AEs were noted and $76 \%$ of patients received the scheduled 3 or 4 cycles of NAC. Dose adjustments were however not investigated due to the retrospective nature of the study.

We did investigate potential risk factors for AEs. The only statistically significant finding was correlation to NAC cycles. This discovery suggests, that if the patient does not tolerate NAC, lack of tolerance manifests already during or after the first cycle. Since most of grade 4 AEs were either hematological $(33 \%)$ or vascular $(30 \%)$, and the single NAC associated death was due to cardiac arrest, patients with symptomatic cardio-vascular diseases may be in the most significant risk. Upfront surgery may be a more viable option in this risk group, since severe AEs may preclude execution of RC. Interestingly scores estimating general comorbidity (ASA score and Charlson comorbidity index) did not demonstrate any correlation with AEs. This demonstrates the difficulty of selecting optimal patients for NAC.

The major limitation of the study is its retrospective nature. However, using this approach we were able to evaluate the incidence of AEs in a real life cohort. Although the number of patients can be considered relatively low, it represents comprehensive multi-institutional national Finnish cohort of patients who received NAC for MIBC between 2008 and 2015. Since the majority of patients received $C G$ as a NAC regimen, the results should not be generalized to cover AEs profiles of different regimens.

\section{CONCLUSION}

This is the first study to report "real life" results in NAC related AEs prior RC. In MIBC patients, NAC is generally well tolerated but poses still a considerable risk of AEs. Patient selection is of critical value to minimize the harmful effect of NAC, which in worst case, may prevent the execution of RC.

\section{AUTHOR CONTRIBUTIONS}

Dr. Antti Salminen gathered the data, designed the study and wrote the manuscript.

Mrs. Ileana Montoya Perez gathered the data, designed the study and edited the manuscript.

Mr. Riku Klén analyzed the data and wrote the manuscript.

Dr. Otto O. Ettala analyzed the data and edited the manuscript.

Dr. Kari T. Syvänen edited the manuscript and supervised the study.

Prof. Laura Elo edited the manuscript and supervised the study.

Dr. Peter J. Boström designed the study, edited the manuscript and supervised the study.

\section{DISCLOSURE}

None of the authors has a conflict of interest related to writing and publishing this manuscript.

\section{ETHICAL CONSIDERATIONS}

The study was approved by the ethical committee of the hospital district of southwestern Finland (ETMK: 4/1802/2015).

\section{ACKNOWLEDGMENTS}

The authors have no acknowledgements.

\section{FUNDING}

The study was funded by a personal grant (Dr. Antti Salminen) by the Finnish Urological association.

\section{COLLABORATORS}

Ilmari Koskinen and Jukka Sairanen, Department of Urology, University of Helsinki and Helsinki University Hospital; Ileana Montoya Perez, Department of Information Technology, University of Turku; Teemu J. Murtola and Petri Virtanen, Department of Urology, University of Tampere and Tampere University Hospital; Markku H. Vaarala and Venla Syri, Department of Urology, University of Oulu and Oulu 
University Hospital; Timo K. Nykopp, Department of Urology, University of Eastern Finland and Kuopio University Hospital; Marjo Seppänen, Department of Surgery, Division of Urology, Central Hospital of Pori; Taina Isotalo, Department of Surgery, Division of Urology, Central Hospital of Lahti; Timo Marttila and Samuli Virtanen, Department of Surgery, Division of Urology, Central Hospital of Seinäjoki; Lasse Levomäki, Department of Surgery, Division of Urology, Central Hospital of Jyväskylä; Sebastian Becker, Department of Surgery, Division of Urology, Central Hospital of Lappeenranta; Mikael Anttinen and Tapani Liukkonen, Department of Surgery, Division of Urology, Central Hospital of Mikkeli; Matti Säily, Department of Surgery, Division of Urology, Central Hospital of Rovaniemi; Dimitri PogodinHannolainen, Department of Surgery, Division of Urology, Central Hospital of Hämeenlinna; Jouko Viitanen, Department of Surgery, Division of Urology, Central Hospital of Joensuu; Christian Palmberg, Department of Surgery, Division of Urology, Central Hospital of Vaasa; Juhani Ottelin, Department of Surgery, Division of Urology, Central Hospital of Kemi.

\section{CONFLICT OF INTEREST}

Antti Salminen reports a personal grant by the Finnish Urological association and a lecturing fee by Roche.

Ileana Montoya Perez, Riku Klén, Otto Ettala and Kari Syvänen report no conflict of interest.

Laura Elo reports grants from the European Research Council ERC (677943), European Union's Horizon 2020 research and innovation programme (675395), Academy of Finland (296801, 304995, 310561 and 313343), Juvenile Diabetes Research Foundation JDRF (2-2013-32), and Sigrid Juselius Foundation, during the conduct of the study. Our research is also supported by University of Turku, Åbo Akademi University, Turku Graduate School (UTUGS), Biocenter Finland, and ELIXIR Finland.

Peter Boström report research grant from Juselius Foundation and Cancer Foundation Finland. Additionally, consultation fees from Astellas and Janssen are reported.

\section{REFERENCES}

[1] Alfred Witjes J, Lebret T, Comperat EM, et al. Updated 2016 EAU guidelines on muscle-invasive and metastatic bladder cancer. Eur Urol. 2017;71:462-75.
[2] Grossman HB, Natale RB, Tangen CM, et al. Neoadjuvant chemotherapy plus cystectomy compared with cystectomy alone for locally advanced bladder cancer. N Engl J Med. 2003;349:859-66.

[3] Chang SS, Bochner BH, Chou R, et al. Treatment of non-metastatic muscle-invasive bladder cancer: AUA/ASCO/ASTRO/SUO guideline. J Urol. 2017; 198:552-9.

[4] Gandaglia G, Popa I, Abdollah F, et al. The effect of neoadjuvant chemotherapy on perioperative outcomes in patients who have bladder cancer treated with radical cystectomy: A population-based study. Eur Urol. 2014;66:561-8.

[5] Johnson DC, Nielsen ME, Matthews J, et al. Neoadjuvant chemotherapy for bladder cancer does not increase risk of perioperative morbidity. BJU Int. 2014;114:221-8.

[6] Salminen AP, Koskinen I, Montoya-Perez I, Hurme S, et al. Neoadjuvant chemotherapy does not increase the morbidity of radical cystectomy: A 10-year retrospective nationwide study. Eur Urol Onc. 2018;6:525-30.

[7] Oun R, Moussa YE, Wheate NJ. The side effects of platinum-based chemotherapy drugs: A review for chemists. Dalton Trans. 2018;47:6645-53.

[8] Duivenvoorden WC, Daneshmand S, Canter D, et al. Incidence, characteristics and implications of thromboembolic events in patients with muscle invasive urothelial carcinoma of the bladder undergoing neoadjuvant chemotherapy. J Urol. 2016;196:1627-33.

[9] Kitamura H, Tsukamoto T, Shibata T, et al. Randomised phase III study of neoadjuvant chemotherapy with methotrexate, doxorubicin, vinblastine and cisplatin followed by radical cystectomy compared with radical cystectomy alone for muscle-invasive bladder cancer: Japan Clinical Oncology Group Study JCOG0209. Ann Oncol. 2014;25:1192-8.

[10] Neoadjuvant cisplatin, methotrexate, and vinblastine chemotherapy for muscle-invasive bladder cancer: A randomised controlled trial. International collaboration of trialists. Lancet. 1999;354:533-40.

[11] Iyer G, Balar AV, Milowsky MI, et al. Multicenter prospective phase II trial of neoadjuvant dose-dense gemcitabine plus cisplatin in patients with muscle-invasive bladder cancer. J Clin Oncol. 2018;36:1949-56.

[12] Common terminology criteria for adverse events v.5 2018 https://ctep.cancer.gov/protocoldevelopment/.../ctcae_v5_ quick_reference_5x7.pdf

[13] Benjamini Y, Hochberg Y. Controlling the false discovery rate: A practical and powerful approach to multiple testing. Journal of the Royal Statistical Society, Series B. 1995;57(1):289-300.

[14] R Core Team. R: A language and environment for statistical computing. R Foundation for Statistical Computing, Vienna, Austria, 2017. URL https://www.R-project.org/

[15] Plimack ER, Hoffman-Censits JH, Viterbo R, et al. Accelerated methotrexate, vinblastine, doxorubicin, and cisplatin is safe, effective, and efficient neoadjuvant treatment for muscle-invasive bladder cancer: Results of a multicenter phase II study with molecular correlates of response and toxicity. J Clin Oncol. 2014;32: 1895-901.

[16] Fairey AS, Daneshmand S, Quinn D, et al. Neoadjuvant chemotherapy with gemcitabine/cisplatin vs. methotrexate/vinblastine/doxorubicin/cisplatin for muscle-invasive urothelial carcinoma of the bladder: A retrospective analysis from the University of Southern California. Urol Oncol. 2013;31:1737-43. 
[17] Galsky MD, Pal SK, Chowdhury S, et al. Comparative effectiveness of gemcitabine plus cisplatin versus methotrexate, vinblastine, doxorubicin, plus cisplatin as neoadjuvant therapy for muscle-invasive bladder cancer. Cancer. 2015;121:2586-93.

[18] von der Maase H, Hansen SW, Roberts JT, et al. Gemcitabine and cisplatin versus methotrexate, vinblastine, doxorubicin, and cisplatin in advanced or metastatic bladder cancer: Results of a large, randomized, multinational, multicenter, phase III study. J Clin Oncol. 2000;18:3068-77.
[19] Dash A, Galsky MD, Vickers AJ, et al. Impact of renal impairment on eligibility for adjuvant cisplatin-based chemotherapy in patients with urothelial carcinoma of the bladder. Cancer. 2006;107:506-13.

[20] Anari F, O'Neill J, Choi W, et al. Neoadjuvant dose-dense gemcitabine and cisplatin in muscle-invasive bladder cancer: Results of a phase 2 trial. Eur Urol Oncol. 2018;1:54-60. 